\title{
Security properties of two authenticated conference key agreement protocols
}

\author{
Qiang Tang and Chris J. Mitchell \\ Information Security Group \\ Royal Holloway, University of London \\ Egham, Surrey TW20 0EX, UK \\ \{qiang.tang, c.mitchell\}@rhul.ac.uk
}

25th June 2005

\begin{abstract}
In this paper we analyse the security of two authenticated group key agreement schemes based on the group key agreement protocol of Burmester and Desmedt. One scheme was proposed by Burmester and Desmedt, and uses a separate authentication scheme to achieve authentication among the participants. We show that this scheme suffers from a number of security vulnerabilities. The other scheme was generated using the general protocol compiler of Katz and Yung. We show that in some circumstances, even if key confirmation is implemented, this scheme still suffers from insider attacks (which are not covered by the security model used by Katz and Yung).
\end{abstract}

\section{Introduction}

Since the pioneering work of Diffie and Hellman [9], key agreement has become a very active and fruitful research area in cryptography. The case of two-party key agreement has been well investigated, and a variety of provably secure schemes have been proposed (e.g., $[1,5,7]$ ). However, less attention has been given to group key agreement, which enables more than two participants to negotiate a session key. Of especial interest are authenticated group key agreement protocols, designed for use in a hostile environment where communications are over open networks which may be fully controlled by an adversary.

Of the existing group key agreement protocols, a number are based on the idea of extending the two-party Diffie-Hellman protocol [9] to the group 
setting (e.g., $[3,12,15,16,17,18]$ ). Among these schemes, the cyclic group key agreement protocol due to Burmester and Desmedt (here referred to as the BD scheme) is particularly efficient; it has been rigorously proved to be secure against a passive adversary [4]. A number of authenticated group key agreement schemes based on the BD scheme have been proposed, including those in $[3,8,10,11,14,19]$. In this paper, we focus on the enhanced BD scheme [3] and a scheme due to Katz and Yung [14], referred to below as the KY scheme.

In the enhanced BD scheme, an interactive zero-knowledge proof scheme is used to achieve authentication among the conference participants, while in the KY scheme a signature mechanism is used to achieve authentication among the conference participants. Both schemes are more efficient than the scheme of Bresson et al. [2], which was the first authenticated group key agreement scheme proved to be secure in a formal model.

The main contribution of this paper lies in analysing the security properties of the BD scheme, and exhibiting potential security vulnerabilities in the enhanced BD scheme and the KY scheme. The rest of this paper is organised as follows. In section 2, we review three group key agreement schemes, namely the BD scheme, the enhanced BD scheme, and the KY scheme. In section 3 , we give our observations on the security of these three schemes. In section 4 , we conclude this paper.

\section{Review of the target schemes}

In all three schemes, the following parameters are made public during the initialisation stage: $G$ is a multiplicative group with large prime order $q$, and $g$ is a generator of $G$. We suppose all the potential participants and their identities are from the set $\left\{\left(U_{1}, I D_{U_{1}}\right), \cdots,\left(U_{m}, I D_{U_{m}}\right)\right\}$, where $m$ is a sufficiently large integer and $I D_{U_{i}}(1 \leq i \leq n \leq m)$ is the identity of $U_{i}$.

\subsection{Description of the BD scheme}

Suppose a set $S=\left\{U_{1}, \cdots, U_{n}\right\}(n \leq m)$ of users wish to establish a session key; then each user $U_{i}(1 \leq i \leq n)$ performs the following steps. It should be noted that the indices of users (and values exchanged between users) are taken modulo $n$.

1. $U_{i}$ chooses a random $s_{i}\left(0 \leq s_{i}<q\right)$, and broadcasts $Z_{i}=g^{s_{i}}$.

2. After receiving $Z_{i-1}$ and $Z_{i+1}, U_{i}$ computes and broadcasts $X_{i}$ :

$$
X_{i}=\left(Z_{i+1} / Z_{i-1}\right)^{s_{i}}
$$


3. After receiving every $X_{j}(1 \leq j \leq n), U_{i}$ computes the session key $K_{i}$ as:

$$
\begin{aligned}
K_{i} & =\left(Z_{i-1}\right)^{n s_{i}} \cdot\left(X_{i}\right)^{n-1} \cdot\left(X_{i+1}\right)^{n-2} \cdots X_{i+n-2} \\
& =g^{n s_{i-1} s_{i}} \cdot\left(\frac{g^{s_{i} s_{i+1}}}{g^{s_{i-1} s_{i}}}\right)^{n-1} \cdot\left(\frac{g^{s_{i+1} s_{i+2}}}{g^{s_{i} s_{i+1}}}\right)^{n-2} \cdots \frac{g^{s_{i+n-2} s_{i+n-1}}}{g^{s_{i+n-3} s_{i+n-2}}} \\
& =g^{s_{i-1} s_{i}+s_{i} s_{i+1}+s_{i+1} s_{i+2}+\cdots+s_{i+n-2} s_{i+n-1}} \\
& =g^{s_{1} s_{2}+s_{2} s_{3}+s_{3} s_{4}+\cdots+s_{n} s_{1}}
\end{aligned}
$$

If all the participants are honest, then all of them will compute the same session key because $K_{1}=\cdots=K_{n}$. In [4], Burmester and Desmedt prove that this scheme is secure against a passive adversary.

\subsection{Description of the enhanced BD scheme}

The enhanced BD scheme provides partial authentication for the protocol messages by using an authentication scheme which is secure against adaptive chosen text attacks. The authentication scheme and the enhanced BD scheme operate as follows.

\subsubsection{The authentication scheme}

In the initialisation stage, the system selects four large primes $p_{2}, p_{3}, q_{2}, q_{3}$ satisfying $p_{2} \leq q_{3}, q_{2} \mid\left(p_{2}-1\right)$, and $q_{3} \mid\left(p_{3}-1\right)$. Let $g_{2}$ be a generator of a multiplicative group of order $q_{2}$ in $Z_{p_{2}}^{*}$, and $g_{3}$ be a generator of a multiplicative group of order $q_{3}$ in $Z_{p_{3}}^{*}$. Each user $U_{i}(1 \leq i \leq n)$ in the system publishes his public key $\left\{\beta_{i 2}, \beta_{i 3}, \gamma_{i 3}\right\}$, where $\left\{\beta_{i 2}=g_{2}^{a_{i 2}}, \beta_{i 3}=g_{3}^{a_{i 3}}, \gamma_{i 3}=g_{3}^{b_{i 3}}\right\}$, and keeps $\left\{a_{i 2}, a_{i 3}, b_{i 3}\right\}$ secret as his private key.

Suppose $U_{i}$ wishes to prove knowledge of $z$ to $U_{j}(j \neq i)$; the authentication scheme operates as follows. $U_{i}$ sends $z$ and $\gamma_{i 2}=g_{2}^{b_{i 2}}$ to $U_{j}$, where $b_{i 2}$ is randomly selected $\left(0 \leq b_{i 2} \leq g_{2}\right)$. Simultaneously $U_{i}$ proves to $U_{j}$ that he knows the discrete logarithm base $g_{2}$ of $\beta_{i 2}^{z} \gamma_{i 2}$ and the discrete logarithm base $g_{3}$ of $\beta_{i 3}^{\gamma_{i 2}} \gamma_{i 3}$, using the zero-knowledge discrete logarithm proof scheme of Chaum et al. [6], described below. $U_{j}$ checks that $\gamma_{i 2}^{q_{2}} \equiv 1\left(\bmod p_{2}\right)$, $g_{2}^{q_{2}} \equiv \beta_{i 2}^{q_{2}} \equiv 1\left(\bmod p_{2}\right), g_{3}^{q_{3}} \equiv \beta_{i 3}^{q_{3}} \equiv \gamma_{i 3}^{q_{3}}\left(\bmod p_{3}\right)$, that $q_{2}, q_{3}$ are primes, and that $p_{2} \leq q_{3}$. If any of the checks fail, $U_{j}$ terminates the protocol. Otherwise $U_{j}$ now believes that $U_{i}$ knows $z$.

The Chaum et al. zero knowledge discrete logarithm proof scheme [6] operates as follows. Suppose $P$ is a large prime, and that $\alpha^{x} \equiv \beta(\bmod P)$. Suppose also that $P, \alpha, \beta$ are made public and $x$ is a secret of Alice. If Alice wants to prove her knowledge of $x$ to Bob, she performs the following steps. 
1. Alice selects $T$ random numbers $e_{i}\left(0 \leq e_{i}<P-1,1 \leq i \leq T\right)$. Alice computes and sends $h_{i}=\alpha^{e_{i}} \bmod P(1 \leq i \leq T)$ to Bob.

2. Bob chooses and sends $T$ random bits $b_{i} \in\{0,1\}(1 \leq i \leq T)$ to Alice.

3. For each bit $b_{i}(1 \leq i \leq T)$, if $b_{i}=0$ Alice sets $s_{i}=e_{i}$; otherwise Alice computes $s_{i}=e_{i}-e_{j} \bmod P-1$, where $j$ is the minimal number for which $b_{j}=1$. Finally, Alice sends $\left(x-e_{j}\right) \bmod P-1$ and $s_{i}$ $(1 \leq i \leq T)$ to Bob.

4. For each bit $i(1 \leq i \leq T)$, if $b_{i}=0$ Bob checks $\alpha^{s_{i}}=h_{i}$; otherwise Bob checks that $\alpha^{s_{i}}=h_{i} h_{j}^{-1}$. Then Bob checks $\alpha^{x-e_{j}}=\beta h_{j}^{-1}$.

If all the checks succeed, Bob can confirm with a probability of $1-\left(\frac{1}{2}\right)^{T}$ that Alice knows $x[6]$.

Burmester and Desmedt [3] claim that the above scheme is a secure authentication system, i.e., it has the following three security properties:

1. When only a passive adversary is present, $U_{i}$ can successfully prove his knowledge of $z$ to $U_{j}$ with an overwhelming probability.

2. If an attacker impersonates $U_{i}$, then $U_{j}$ can detect it with an overwhelming probability.

3. If an active attacker substitutes $z$ with $z^{\prime}\left(z \neq z^{\prime}\right)$, then $U_{j}$ will reject it with an overwhelming probability.

\subsubsection{The enhanced BD scheme}

Suppose a set $S=\left\{U_{1}, \cdots, U_{n}\right\}(n \leq m)$ of users wish to establish a session key; then each user $U_{i}(1 \leq i \leq n)$ performs the following steps. Note that the indices of users (and values exchanged between users) are taken modulo $n$.

1. $U_{i}$ chooses a random $s_{i}\left(0 \leq s_{i} \leq q\right)$, and computes and broadcasts $Z_{i}=g^{s_{i}}$.

2. After receiving $Z_{i-1}$ and $Z_{i+1}, U_{i}$ proves his knowledge of $s_{i}$ to $U_{i+1}$, and verifies $U_{i-1}$ 's knowledge of $s_{i-1}$.

If both the proof and the verification succeed, $U_{i}$ computes and broadcasts $X_{i}$ :

$$
X_{i}=\left(Z_{i+1} / Z_{i-1}\right)^{s_{i}}
$$


3. After receiving $X_{j}(1 \leq j \leq n), U_{i}$ computes the session key $K_{i}$ :

$$
\begin{aligned}
K_{i} & =\left(Z_{i-1}\right)^{n s_{i}} \cdot\left(X_{i}\right)^{n-1} \cdot\left(X_{i+1}\right)^{n-2} \cdots X_{i+n-2} \\
& =g^{n s_{i-1} s_{i}} \cdot\left(\frac{g^{s_{i} s_{i+1}}}{g^{s_{i-1} s_{i}}}\right)^{n-1} \cdot\left(\frac{g^{s_{i+1} s_{i+2}}}{g^{s_{i} s_{i+1}}}\right)^{n-2} \cdots \frac{g^{s_{i+n-2} s_{i+n-1}}}{g^{s_{i+n-3} s_{i+n-2}}} \\
& =g^{s_{i-1} s_{i}+s_{i} s_{i+1}+s_{i+1} s_{i+2}+\cdots+s_{i+n-2} s_{i+n-1}} \\
& =g^{s_{1} s_{2}+s_{2} s_{3}+s_{3} s_{4}+\cdots+s_{n} s_{1}}
\end{aligned}
$$

If all the participants are honest, then all of them will compute the same session key because $K_{1}=\cdots=K_{n}$.

Burmester and Desmedt [3] claim that the enhanced BD scheme is a secure key agreement scheme, i.e., in a protocol instance it is computationally infeasible for any set of active attackers to compute the same session key as that which is computed by the honest participants.

\subsection{Description of the KY scheme}

Katz and Yung [14] proposed a general protocol compiler that can transform a group key agreement protocol secure against a passive adversary into an authenticated group key agreement protocol secure against both passive and active adversaries. As an example, Katz and Yung transformed the unauthenticated BD scheme into an authenticated group key agreement protocol. Katz and Yung [14] prove that this protocol is secure against an active adversary, i.e., the advantage of any probabilistic polynomial time (PPT) active adversary is negligible.

The KY scheme [14] requires that, during the initialisation stage, each user $U_{i}(1 \leq i \leq m)$ generates a verification/signing key pair $\left(P K_{U_{i}}, S K_{U_{i}}\right)$ by running $\operatorname{Gen}\left(1^{k}\right)^{1}$, where $k$ is a security parameter. Suppose a set $S=\left\{U_{1}, \cdots, U_{n}\right\}(n \leq m)$ of users wish to establish a session key; then each user $U_{i}(1 \leq i \leq n)$ performs the following steps. It should be noted that, as previously, the indices of users (and values exchanged between users) are taken modulo $n$. Throughout this paper, || represents the string concatenation operator.

1. $U_{i}$ chooses a random $r_{i}\left(0 \leq r_{i}<q\right)$ and broadcasts $I D_{U_{i}}, 0$, and $r_{i}$.

2. After receiving the broadcast messages from all other participants, $U_{i}$ sets nonce $_{i}=\left(\left(I D_{U_{1}}, r_{1}\right), \cdots,\left(I D_{U_{n}}, r_{n}\right)\right)$ and stores it as part of its state information ${ }^{2}$

\footnotetext{
${ }^{1}$ We suppose that $\Sigma=($ Gen, Sign, Vrfy $)$ is a signature scheme which is strongly unforgeable under adaptive chosen message attack (as defined in [14]).

${ }^{2}$ If all the messages are transported correctly, every user will possess the same state information.
} 
$U_{i}$ chooses a random number $s_{i}\left(0 \leq s_{i}<q\right)$ and computes $Z_{i}=g^{s_{i}}$. Then $U_{i}$ computes the signature $\sigma_{i 1}=\operatorname{Sign}_{S K_{U_{i}}}\left(1\left\|Z_{i}\right\|\right.$ nonce $\left._{i}\right)$ and broadcasts $I D_{U_{i}}, 1, Z_{i}$, and $\sigma_{i 1}$.

3. When $U_{i}$ receives the message $I D_{U_{j}}, 1, Z_{j}$, and $\sigma_{j 1}$ from user $U_{j}$ $(1 \leq j \leq n, j \neq i)$, he checks that: (1) $U_{j}$ is an intended participant,

(2) 1 is the next expected sequence number for a message from $U_{j}$, and

(3) $\operatorname{Vrfy}_{P K_{U_{j}}}\left(1\left\|Z_{j}\right\|\right.$ nonce $\left._{i}, \sigma_{j 1}\right)=1$, where an output of 1 signifies acceptance. If any of these checks fail, $U_{i}$ terminates the protocol. Otherwise, $U_{i}$ computes $X_{i}=\left(Z_{i+1} / Z_{i-1}\right)^{s_{i}}$ and the signature $\sigma_{i 2}=$ $\operatorname{Sign}_{S K_{U_{i}}}\left(2\left\|X_{i}\right\|\right.$ nonce $\left._{i}\right)$. Then $U_{i}$ broadcasts $I D_{U_{i}}, 2, X_{i}$, and $\sigma_{i 2}$.

4. When $U_{i}$ receives the message $I D_{U_{j}}, 2, X_{j}$, and $\sigma_{j 2}$ from user $U_{j}$ $(1 \leq j \leq n, j \neq i)$, he checks that: (1) $U_{j}$ is an intended participant, (2) 2 is the next expected sequence number for a message from $U_{j}$, and (3) $\operatorname{Vrfy}_{P K_{U_{j}}}\left(2|| X_{j} \|\right.$ nonce $\left._{i}, \sigma_{j 2}\right)=1$. If any of these checks fail, $U_{i}$ terminates the protocol. Then $U_{i}$ computes the session key $K_{i}$ :

$$
\begin{aligned}
K_{i} & =\left(Z_{i-1}\right)^{n s_{i}} \cdot\left(X_{i}\right)^{n-1} \cdot\left(X_{i+1}\right)^{n-2} \cdots X_{i+n-2} \\
& =g^{n s_{i-1} s_{i}} \cdot\left(\frac{g^{s_{i} s_{i+1}}}{g^{s_{i-1} s_{i}}}\right)^{n-1} \cdot\left(\frac{g^{s_{i+1} s_{i+2}}}{g^{s_{i} s_{i+1}}}\right)^{n-2} \cdots \frac{g^{s_{i+n-2} s_{i+n-1}}}{g^{s_{i+n-3} s_{i+n-2}}} \\
& =g^{s_{i-1} s_{i}+s_{i} s_{i+1}+s_{i+1} s_{i+2}+\cdots+s_{i+n-2} s_{i+n-1}} \\
& =g^{s_{1} s_{2}+s_{2} s_{3}+s_{3} s_{4}+\cdots+s_{n} s_{1}}
\end{aligned}
$$

If all the participants are honest, then all of them will compute the same session key $K=K_{1}=\cdots=K_{n}=g^{s_{1} s_{2}+s_{2} s_{3}+\cdots+s_{n} s_{1}}$.

Katz and Yung [14] also proposed the following method (without security proof) to achieve key confirmation for the authenticated group key agreement scheme: after computing key $K$, each player $U_{i}$ computes $x_{i}=$ $F_{K}\left(I D_{U_{i}}\right)$, signs $x_{i}$, and broadcasts $x_{i}$ and the corresponding signature, where $F$ represents a pseudo-random function. However, they did not specify how the signature is computed. In this paper, we suppose the signature is computed as $\sigma_{i j}=\operatorname{Sign}_{S K_{U_{i}}}\left(x_{i}\right)$, where $j$ is the next round number.

However, it should be noted that a different key confirmation method is proposed in the full version of [14], and this method is proved to be secure in a different security model in [13].

\section{Properties of the target schemes}

In this section we first describe certain security properties of the BD scheme, and then demonstrate security vulnerabilities in both the enhanced BD scheme and the KY scheme. 


\subsection{Security properties of the BD scheme}

In the $\mathrm{BD}$ scheme, a malicious participant, say $U_{j}(1 \leq j \leq n)$, who can manipulate the communications in the network, is able to make any other participant, say $U_{i}(1 \leq i \leq n, i \neq j)$, compute the session key to be any value $K^{*} \in G$ chosen by $U_{j}$.

To achieve this, in the second step $U_{j}$ intercepts the message $X_{i-n+2}$ and prevents it from reaching $U_{i}$. $U_{j}$ then waits until all the other messages have been received and computes the session key $K$ in the normal way, i.e. as in step 3 of section 2.1. $U_{j}$ now sends $X_{i+n-2}^{\prime}=X_{i+n-2} \cdot \frac{K^{*}}{K}$ to $U_{i}$, pretending that it comes from $U_{i+n-2}$.

Lemma 3.1. As a result of the above attack, $U_{i}$ will compute the session key as $K^{*}$.

Proof. This is immediate, since $U_{i}$ will compute the session key as $K$. $\frac{X_{i+n-2}^{\prime}}{X_{i+n-2}}=K^{*}$, by definition of $X_{i+n-2}^{\prime}$.

In summary, in the BD scheme any participant capable of manipulating the messages received by another participant can completely control the value of the session key obtained by that participant. In the following subsections, we show that this property means that schemes derived from the BD scheme possess certain security vulnerabilities.

\subsection{Security vulnerabilities in the enhanced BD scheme}

The enhanced BD scheme suffers from the following potential security vulnerabilities.

\subsubsection{Man-in-the-middle attack}

To mount a man-in-the-middle attack, an active adversary proceeds as follows. In the first step of the protocol the adversary replaces the message $Z_{i+1}$ sent to $U_{i}$ with $Z_{i+1}^{\prime}=Z_{i-1}^{2}$, for every $i(1 \leq i \leq n)$. Then we can prove that the protocol will end successfully and the adversary can compute the session key held by $U_{i}(1 \leq i \leq n)$.

Lemma 3.2. Under the above attack, the protocol will end successfully, and the adversary can compute the session key held by $U_{i}$ for every $i(1 \leq i \leq n)$.

Proof. Under the attack, it is clear that the protocol will end successfully, because it is only required that $U_{i}(1 \leq i \leq n)$ proves his knowledge of $s_{i}$ to $U_{i+1}$ (while the adversary only changes the message that $U_{i+1}$ sends to $U_{i}$ ). 
It is also clear that, in the second step, $U_{i}$ will broadcast $X_{i}=\left(Z_{i+1}^{\prime} / Z_{i-1}\right)^{s_{i}}=$ $\left(Z_{i-1}\right)^{s_{i}}$. Then, after intercepting all the broadcast values $X_{i}(1 \leq i \leq n)$, the adversary can compute the session key held by $U_{i}$ as

$$
\begin{aligned}
K_{i} & =\left(Z_{i-1}\right)^{n s_{i}} \cdot\left(X_{i}\right)^{n-1} \cdot\left(X_{i+1}\right)^{n-2} \cdots X_{i+n-2} \\
& =\left(X_{i}\right)^{n} \cdot\left(X_{i}\right)^{n-1} \cdot\left(X_{i+1}\right)^{n-2} \cdots X_{i+n-2}
\end{aligned}
$$

which involves only values broadcast by the various recipients. The result follows.

\subsubsection{Insider different key attack}

In the enhanced $\mathrm{BD}$ scheme, it is only required that $U_{i}(1 \leq i \leq n)$ proves his knowledge of $s_{i}$ to $U_{i+1}$. The authentication requirement can successfully prevent a malicious attacker from impersonating $U_{i}(1 \leq i \leq n)$ to send a forged message $Z_{i}$ to the honest participant $U_{i+1}$. However, a malicious participant, say $U_{j}(1 \leq j \leq n)$, who can manipulate the communications in the network, is still able to make any other participant, say $U_{i}(1 \leq i \leq$ $n, i \neq j$ ), compute the session key to be any value $K^{*} \in G$.

In fact, any active outsider attacker can also mount such an attack by manipulating $X_{i}(1 \leq i \leq n)$ in step 2 of the protocol run, but the attacker cannot obtain any information about the session keys obtained by the legitimate participants.

\subsubsection{Outsider impersonation attack}

An outsider attacker can also impersonate a valid participant, $U_{i}$ say, in some circumstances, but the attacker cannot obtain the session key. This attack is based on the following security vulnerability in the Chaum et al. zero-knowledge discrete logarithm proof scheme ${ }^{3}$. The vulnerability arises from the fact that proof scheme does not enable the prover to specify the verifier.

Suppose Alice wishes to prove her knowledge of $x$ to Bob, then an attacker can concurrently impersonate Alice to prove knowledge of $x$ to any other entity, Carol say. The attack can be mounted as follows.

1. Alice selects $T$ random numbers $e_{i}\left(0 \leq e_{i} \leq P-1,1 \leq i \leq T\right)$, and computes and sends $h_{i}=\alpha^{e_{i}} \bmod P(1 \leq i \leq T)$ to Bob.

The attacker intercepts the message, prevents it from reaching Bob, and forwards $h_{i}=\alpha^{e_{i}} \bmod P(1 \leq i \leq T)$ to Carol pretending to be Alice (i.e. starting a second run of the protocol).

\footnotetext{
${ }^{3}$ It should be noted that this vulnerability exists not only in this specific scheme, but also exists in all such schemes with only a one-way proof of knowledge.
} 
2. Carol chooses and sends random bits $e_{i} \in\{0,1\}(1 \leq i \leq T)$ to the attacker as part of the second protocol run. The attacker then impersonates Bob to forward $e_{i} \in\{0,1\}(1 \leq i \leq T)$ to Alice as the second message of the first protocol run.

3. For each bit $b_{i}(1 \leq i \leq T)$, if $b_{i}=0$ Alice sets $s_{i}=e_{i}$; otherwise Alice computes $s_{i}=e_{i}-e_{j} \bmod P-1$, where $j$ is the minimal number that $b_{j}=1$. Alice sends $x-e_{j}$ and $s_{i}(1 \leq i \leq T)$ to Bob as the third message of the first protocol run. The attacker intercepts the message, prevents it from reaching Bob, and forwards it to Carol as the third message of the second protocol run.

4. For each bit $i(1 \leq i \leq T)$, if $b_{i}=0$ Carol checks $\alpha^{s_{i}}=h_{i}$; otherwise Carol checks that $\alpha^{s_{i}}=h_{i} h_{j}^{-1}$. Then Carol checks that $\alpha^{x-e_{j}}=\beta h_{j}^{-1}$.

It is easy to verify that Carol's checks will succeed and confirm that the attacker knows $x$. This attack conflicts with the claim made in [3] that the authentication technique is secure against any type of attack.

We now show how to use the above observation to attack the enhanced BD scheme. Suppose the attacker detects that a set $S=\left\{U_{1}, \cdots, U_{n}\right\}$ of users is starting the key agreement protocol to negotiate a session key (referred to below as the first protocol instance). The attacker impersonates $U_{i}$ to initiate a second instance of the key agreement protocol among a different set $S^{\prime}=\left\{U_{1}^{\prime}, U_{2}^{\prime}, \ldots, U_{n^{\prime}}^{\prime}\right\}$ of users, where $U_{i}^{\prime}=U_{i}$. In these two protocol instances, the attacker performs the following steps.

1. In the first protocol instance, $U_{i}$ chooses a random $s_{i}\left(0 \leq s_{i}<q\right)$, and computes and broadcasts $Z_{i}=g^{s_{i}}$. The attacker intercepts the messages from both $U_{i-1}$ and $U_{i+1}$ to $U_{i}$ and prevents them from reaching $U_{i}$.

In the second protocol instance, the attacker impersonates $U_{i}$ to broadcast $Z_{i}=g^{s_{i}}$. Other participants perform as required by the protocol. Suppose the messages sent by $U_{i-1}^{\prime}$ and $U_{i+1}^{\prime}$ are $Z_{i-1}^{\prime}$ and $Z_{i+1}^{\prime}$.

The attacker impersonates $U_{i-1}$ and $U_{i+1}$ to send $Z_{i-1}^{\prime}$ and $Z_{i+1}^{\prime}$ to $U_{i}$ in the first protocol instance.

2. In the first protocol instance, when $U_{i}$ proves his knowledge of $s_{i}$ to $U_{i+1}$, the attacker mounts the above attack against the Chaum et al. zero-knowledge discrete logarithm proof scheme by impersonating $U_{i}$ to prove his knowledge of $s_{i}$ to $U_{i+1}^{\prime}$ in the second protocol instance.

In the second protocol instance, when $U_{i-1}^{\prime}$ proves his knowledge of $s_{i-1}^{\prime}$ to the attacker, the attacker mounts the above attack against the Chaum et al. zero-knowledge discrete logarithm proof scheme by 
impersonating $U_{i-1}^{\prime}$ to prove $U_{i-1}^{\prime}$ 's knowledge of $s_{i-1}^{\prime}$ to $U_{i}$ in the first protocol instance.

In the first protocol instance $U_{i}$ computes and broadcasts $X_{i}$ as:

$$
X_{i}=\left(Z_{i+1}^{\prime} / Z_{i-1}^{\prime}\right)^{s_{i}}
$$

The attacker intercepts this message and impersonates $U_{i}$ to broadcast the same message in the second protocol instance.

3. In the second protocol instance, the users $U_{j}^{\prime}\left(1 \leq j \leq n^{\prime}, j \neq i\right)$ computes the common session key. However, the attacker cannot compute the session key because he does not know $s_{i}$.

The first instance will be terminated because the authentication messages between $U_{i}$ and $U_{i+1}$ are blocked by the attacker.

In the second protocol instance, the attacker succeeds in impersonating $U_{i}$ to the members of the set $S^{\prime}$.

\subsubsection{Insider impersonation attack}

In the above attack, suppose that the attacker is a legitimate participant in the second protocol instance, i.e. suppose that the attacker is $U_{j}^{\prime}$ in the set $S^{\prime}$ $\left(U_{j}^{\prime}\right.$ is not required to be a member of the set $S$ ). Then $U_{j}^{\prime}$ can successfully impersonate $U_{i}$ in the second protocol instance without any knowledge of the secret key of $U_{i}$. In this case, it is clear that $U_{j}^{\prime}$ can compute the session key agreed by all the participants of $S^{\prime}$, since $U_{j}^{\prime}$ is a legitimate member of $S^{\prime}$.

This attack means that, even if authentication is implemented, a malicious participant can still impersonate another honest participant in a protocol instance.

\subsection{Potential security vulnerability in the KY scheme}

We show that the KY scheme suffers from insider different key attacks even if key confirmation is implemented. Specifically, we show that any $n-$ 2 malicious participants can make the other honest participants compute different keys at the end of the protocol. However, it should be noted that insider attacks are not covered by the security model in [14]. Recently, insider attacks have been modelled by Katz and Shin in [13].

For simplicity we describe the attack in three-party case. Suppose, in some past successful instance of the KY protocol among $\left\{U_{1}, U_{2}, U_{3}\right\}$, the key confirmation message sent by $U_{3}$ is $x_{3}^{*}=F_{K_{3}^{*}}\left(I D_{U_{3}}\right)$ and $\sigma_{33}^{*}=\operatorname{Sign}_{S K_{U_{3}}}\left(x_{3}^{*}\right)$. 
$U_{2}$ can initiate a new instance of the KY protocol among $\left\{U_{1}, U_{2}, U_{3}\right\}$ and mount a different key attack as follows.

1. Each user $U_{i}(1 \leq i \leq 3)$ begins by choosing a random $r_{i}\left(0 \leq r_{i}<q\right)$ and broadcasting $I D_{U_{i}}, 0$, and $r_{i}$.

2. After receiving the initial broadcast messages, $U_{i}(1 \leq i \leq 3)$ sets nonce $_{i}=\left(\left(I D_{U_{1}}, r_{1}\right),\left(I D_{U_{2}}, r_{2}\right),\left(I D_{U_{3}}, r_{3}\right)\right)$ and stores it as part of its state information. Then $U_{i}$ chooses a random $s_{i}\left(0 \leq s_{i}<q\right)$, computes $Z_{i}=g^{s_{i}}$ and the signature $\sigma_{i 1}=\operatorname{Sign}_{S K_{U_{i}}}\left(1\left\|Z_{i}\right\|\right.$ nonce $\left._{i}\right)$, and then broadcasts $I D_{U_{i}}, 1, Z_{i}$, and $\sigma_{i 1}$.

3. When $U_{i}(1 \leq i \leq 3)$ receives the messages from other participants, he checks the messages as required by the protocol. It is easy to verify that all the checks will succeed.

$U_{1}$ computes and then broadcasts $I D_{U_{1}}, 2, X_{1}$, and $\sigma_{12}$, where

$$
X_{1}=\left(Z_{2} / Z_{3}\right)^{s_{1}}, \sigma_{12}=\operatorname{Sign}_{S K_{U_{1}}}\left(2\left\|X_{1}\right\| \text { nonce }_{1}\right) .
$$

$U_{3}$ computes and then broadcasts $I D_{U_{3}}, 2, X_{3}$, and $\sigma_{32}$, where

$$
X_{3}=\left(Z_{1} / Z_{2}\right)^{r_{3}}, \sigma_{32}=\operatorname{Sign}_{S K_{U_{3}}}\left(2\left\|X_{3}\right\| \text { nonce }_{3}\right)
$$

$U_{2}$ computes and sends $I D_{U_{2}}, 2, X_{2}$, and $\sigma_{22}$ to $U_{3}$, where

$$
X_{2}=\left(Z_{3} / Z_{1}\right)^{s_{2}}, \sigma_{22}=\operatorname{Sign}_{S K_{U_{2}}}\left(2\left\|X_{2}\right\| \text { nonce }_{2}\right)
$$

$U_{2}$ then waits until all the other messages have been received and computes the session key $K$ in the normal way, i.e. as in step 3 of section 2.1. $U_{2}$ now sends $I D_{U_{2}}, 2, X_{2}^{\prime}$, and $\sigma_{22}^{\prime}$ to $U_{1}$, where

$$
X_{2}^{\prime}=X_{2} \cdot \frac{K_{3}^{*}}{K}, \sigma_{22}^{\prime}=\operatorname{Sign}_{S K_{U_{2}}}\left(2\left\|X_{2}^{\prime}\right\| \text { nonce }_{2}\right)
$$

Lemma 3.3. As a result of the above steps, $U_{1}$ will compute the session key as $K_{3}^{*}$, and $U_{3}$ will computes the session key as $K$.

Proof. This is immediate, since $U_{1}$ will compute the session key as $\left(Z_{3}\right)^{3 s_{1}}\left(X_{1}\right)^{2} X_{2}^{\prime}=K \cdot \frac{K_{3}^{*}}{K}=K_{3}^{*}$, and $U_{3}$ will compute the session key as $\left(Z_{2}\right)^{3 s_{3}}\left(X_{3}\right)^{2} X_{1}=K$.

Hence, as a result, $U_{2}$ shares the session keys $K_{3}^{*}$ and $K\left(K \neq K_{3}^{*}\right)$ with $U_{1}$ and $U_{3}$ respectively. 
4. $U_{2}$ intercepts the confirmation messages between $U_{1}$ and $U_{3}$ and prevents them from reaching their indeed destinations. $U_{2}$ computes and sends the confirmation messages $x_{2}^{\prime}=F_{K_{3}^{*}}\left(I D_{U_{2}}\right)$ and $\sigma_{23}^{\prime}=$ $\operatorname{Sign}_{S K_{U_{2}}}\left(x_{2}^{\prime}\right)$ to $U_{1}$, and then sends $x_{2}=F_{K}\left(I D_{U_{2}}\right)$ and $\sigma_{23}=$ $\operatorname{Sign}_{S K_{U_{2}}}\left(x_{2}\right)$ to $U_{3}$. Then $U_{2}$ impersonates $U_{3}$ to send $x_{33}^{*}$ and $\sigma_{33}^{*}$ to $U_{1}$.

$U_{2}$ initiates a second instance of the key agreement protocol among the members of a set $S^{\prime \prime}$, which includes $U_{1}$ and $U_{2}$. In step 3 of the new instance, $U_{2}$ manipulates the communications and forces $U_{1}$ to compute the session key as $K$, and then obtains the confirmation message $\left(x_{1}=F_{K}\left(I D_{U_{1}}\right), \sigma_{13}=\operatorname{Sign}_{S K_{U_{1}}}\left(x_{1}\right)\right)$ from $U_{1}$.

$U_{2}$ impersonates $U_{1}$ to forward $\left(x_{1}=F_{K}\left(I D_{U_{1}}\right), \sigma_{13}=\operatorname{Sign}_{S K_{U_{1}}}\left(x_{1}\right)\right)$ to $U_{3}$ in the current protocol instance.

5. It is easy to verify that all the key confirmation messages will be checked successfully by the various participants, and the attack will therefore succeed.

\section{Conclusions}

In this paper we have shown that a number of security vulnerabilities exist in both the enhanced BD scheme and the KY scheme. In particular, we have shown that in the enhanced BD scheme the implementation of the authentication scheme does not meet the authentication requirement specified in [3].

\section{Acknowledgements}

The authors would like to express their deep appreciation for the valuable comments provided by Jonathan Katz and Kenneth G. Paterson.

\section{References}

[1] M. Bellare and P. Rogaway. Entity authentication and key distribution. In D. R. Stinson, editor, Advances in Cryptology - Crypto '93, volume 773 of Lecture Notes in Computer Science, pages 110-125. SpringerVerlag, 1993.

[2] E. Bresson, O. Chevassut, D. Pointcheval, and J.-J. Quisquater. Provably authenticated group Diffie-Hellman key exchange. In Proceedings 
of the 8th ACM Conference on Computer and Communications Security, pages 255-264. ACM Press, 2001.

[3] M. Burmester and Y. Desmedt. A secure and efficient conference key distribution system. In A. D. Santis, editor, Advances in CryptologyEUROCRYPT '94, volume 950 of Lecture Notes in Computer Science, pages 275-286. Springer-Verlag, 1994.

[4] M. Burmester and Y. Desmedt. A secure and efficient conference key distribution system. In A. D. Santis, editor, Pre-Proceedings of Eurocrypt '94, pages 279-290, 1994.

[5] R. Canetti and H. Krawczyk. Analysis of key-exchange protocols and their use for building secure channels. In B. Pfitzmann, editor, Advances in Cryptology - Eurocrypt 2001, volume 2045 of Lecture Notes in Computer Science, pages 453-474. Springer-Verlag, 2001.

[6] D. Chaum, J.-H. Evertse, J. Graaf, and R. Peralta. Demonstrating possession of a discrete logarithm without revealing it. In A. M. Odlyzko, editor, Advances in Cryptology-CRYPTO '86, pages 200212. Springer-Verlag, 1987.

[7] L. Chen and C. Kudla. Identity based authenticated key agreement protocols from pairings. In Proc. of the 16th IEEE Computer Security Foundations Workshop - CSFW 2003, pages 219-233. IEEE Computer Society Press, 2003.

[8] K. Y. Choi, J. Y. Hwang, and D. H. Lee. Efficient ID-based group key agreement with bilinear maps. In F. Bao, R. Deng, and J. Y. Zhou, editors, Proceedings of the 2004 International Workshop on Practice and Theory in Public Key Cryptography (PKC '04), volume 2947 of Lecture Notes in Computer Science, pages 130-144. Springer-Verlag, 2004.

[9] W. Diffie and M. Hellman. New directions in cryptography. IEEE Transactions on Information Theory, IT-22(6):644-654, 1976.

[10] X. J. Du, Y. Wang, J. H. Ge, and Y. M. Wang. ID-based authenticated two round multiparty key agreement. Cryptology ePrint Archive: Report 2003/247, 2003.

[11] X. J. Du, Y. Wang, J. H. Ge, and Y. M. Wang. An improved ID-based authenticated group key agreement scheme. Cryptology ePrint Archive, Report 2003/260, 2003.

[12] I. Ingemarsson, D. Tang, and C. Wong. A conference key distribution system. IEEE Transactions on Information Theory, 28(5):714-720, 1982. 
[13] J. Katz and J. Shin. Modeling insider attacks on group key-exchange protocols. Cryptology ePrint Archive: Report 2005/163, 2005.

[14] J. Katz and M. Yung. Scalable protocols for authenticated group key exchange. In D. Boneh, editor, Advances in Cryptology - Crypto 2003, volume 2729 of Lecture Notes in Computer Science, pages 110-125. Springer-Verlag, 2003.

[15] Y. Kim, A. Perrig, and G. Tsudik. Communication-efficient group key agreement. In Proc. IFIP TC11 16th Annual Working Conference on Information Security, pages 229-244, 2001.

[16] D. Steer, L. Strawczynski, W. Diffie, and M. Wiener. A secure audio teleconference system. In H. Krawczyk, editor, Advances in Cryptology - Crypto '98, volume 403 of Lecture Notes in Computer Science, pages 520-528. Springer-Verlag, 1998.

[17] W. Tzeng. A practical and secure-fault-tolerant conferenc-key agreement protocol. In H. Imai and Y. Zheng, editors, Proceedings of Public Key Cryptography: Third International Workshop on Practice and Theory in Public Key Cryptosystems, pages 1-13. Springer-Verlag, 2000.

[18] W. Tzeng. A secure fault-tolerant conference-key agreement protocol. IEEE Transactions on Computers, 51(4):373-379, 2002.

[19] F. G. Zhang and X. F. Chen. Attacks on two ID-based authenticated group key agreement schemes. Cryptology ePrint Archive, Report 2003/259, 2003. 